\title{
EPISTEMOLOGÍA SOFISTA Y SU INFLUENCIA EN LA TERAPIA BREVE ESTRATÉGICA. MODELO NARDONE
}

\author{
SOPHIST EPISTEMOLOGY AND ITS INFLUENCE ON BRIEF STRATEGIC THERAPY. \\ NARDONE MODEL
}

\author{
Ricardo de la Cruz Gil* \\ Centro de Terapia Breve Estratégica del Perú \\ Lima-Perú \\ Recibido febrero de 2021/Received February, 2021 \\ Aceptado abril de 2021/Accepted April, 2021
}

\begin{abstract}
RESUMEN
El presente artículo pretende realizar una revisión y análisis de la relación e influencia del pensamiento y epistemología sofista en la moderna psicoterapia breve estratégica, modelo de Giorgio Nardone. El interés particular de la investigación gira en torno a los aspectos epistemológicos y el cuestionamiento de una realidad objetiva y cómo esta puede haber influido en la praxis de la terapia breve. Los aportes epistemológicos, teóricos y filosóficos de los sofistas promovieron un cambio cultural en el mundo helénico, su legado llega hasta nuestros días influyendo enormemente en diversos campos del conocimiento, desde la filosofía hasta la psicoterapia. Se presenta una revisión de la evidencia preliminar publicada en la literatura filosófica y psicológica examinando la epistemología sofista y su influencia en la terapia breve estratégica, analizando su enfoque epistemológico relacionado al relativismo y el escepticismo, dando muestras al mismo tiempo de una filosofía que llega hasta nuestros días y que influye en el pensamiento contemporáneo y en la psicoterapia moderna especialmente en la terapia breve estratégica. A partir de la revisión y análisis se evidencia la influencia de los sofistas y su pensamiento en la epistemología, el cuestionamiento de una realidad objetiva y la praxis clínica de la terapia breve estratégica.
\end{abstract}

Palabras Clave: Sofistas, Terapia Breve Estratégica, Epistemología, Psicoterapia.

\section{ABSTRACT}

This article aims to carry out a review and analysis of the relationship and influence of sophist thought and epistemology in modern Brief strategic psychotherapy, Nardone model. The particular interest of this research revolves around the epistemological aspects and the questioning of objective reality and how this may have influenced the praxis of brief therapy. The epistemological, theoretical, and philosophical contributions of the sophists promoted a cultural change in the Hellenic world their legacy continues to this day, greatly influencing various fields of knowledge, from philosophy to psychotherapy. A review of the preliminary evidence published in the philosophical and psychological literature is presented, examining sophist epistemology and its influence on brief strategic therapy, analyzing its epistemological approach related to relativism and skepticism, at the same time showing a philosophy that continues to this day that influences contemporary thinking and modern psychotherapy, especially Brief Strategic Therapy. From the review and analysis, is evidenced the influence of the sophists thinking on epistemology, the questioning of objective reality, and the clinical practice of brief strategic therapy.

Key Words: Sophists, Brief Strategic Therapy, Epistemology, Psychotherapy. 


\section{INTRODUCCIÓN}

La Grecia de la posguerra vencedora de los persas empieza una nueva era de desarrollo social, cultural y económico gracias a la figura de Pericles quien brindó el contexto que permitió el fortalecimiento y desarrollo de la democracia y con ello una mayor participación ciudadana, lo que lleva a la sociedad ateniense a centrar su mirada en la vida social y cultural, deja de pensar en la naturaleza y sus fenómenos para centrarse en temas más relacionados a lo social, a la polis y a la participación pública (Guthrie, 2003). La victoria sobre los persas supone para Grecia y en especial para Atenas el inicio del auge y desarrollo económico, social y cultural, supone también la consolidación de la democracia y con ella cambios en la estructura social, lo que llevó también al desarrollo de la filosofía y un cambio en la política y la democracia, los ciudadanos que contribuyeron con la victoria reclaman una mayor participación en la sociedad ateniense, toda la sociedad ateniense voltea su mirada a la vida pública. La democracia, debido a sus características, permite la participación ciudadana en la vida política, se es exitoso si se participa en el ágora, en la plaza pública, en el debate político, es por ello que muchos comerciantes adinerados desean que sus jóvenes hijos se preparen para participar de la vida pública, ya no se trata de herencia o linaje, la democracia permite la participación abierta de los ciudadanos libres en el gobierno de la ciudad (De Romilly, 1997). Con la consolidación de la democracia ateniense el éxito ahora está relacionado al liderazgo político, a la retórica, a la oratoria, en fin, a las condiciones que todo líder debe de evidenciar para ser reconocido o aclamado por el pueblo, en tanto sea más reconocido o aclamado por las masas en el ágora o plaza pública, será más exitoso, por tanto demostrar las cualidades de un buen orador y convencer a las masas derrotando con sólidos argumentos a sus adversarios son condiciones necesarias para el éxito. En ese contexto tener las competencias y habilidades necesarias para poder triunfar en los debates públicos se hace necesario, saber expresarse es condición muy importante para lograr alcanzar la fama y ostentar el poder en la nueva sociedad ateniense, un éxito más que relacionado al linaje, relacionado ahora a las capacidades, al poder de la palabra, demostrado en la asamblea, por ello las habilidades para el liderazgo, la oratoria y la persuasión son imprescindibles en la nueva etapa que vive la sociedad ateniense, la retórica, la oratoria y la argumentación se han convertido en esenciales para tener éxito en la vida pública y en la política ateniense. Se dan de esta forma las condiciones para el éxito de los sofistas, ya que ellos son expertos en el arte de la retórica y la persuasión, entendida la primera como el arte cívico de la oratoria pública (Álvarez, 2016). Es por ello que los sofistas tienen un gran auge y ganan mucha fama, es en ese contexto que surgen como sabios especializados en el arte de la retórica, la oratoria y la persuasión.

Otro factor importante para analizar y tener en cuenta en el surgimiento de los sofistas, es el sistema educativo ateniense, ya que todo este desarrollo de la democracia y el crecimiento social, económico y cultural llevó también a un auge y fortalecimiento del sistema educativo, el ciudadano común no solo reclama una mayor participación en la vida pública sino que ahora centra su atención en la educación y en el desarrollo de las capacidades, como mecanismo de ascensión y crecimiento social (Guthrie, 2003). Recordemos que este ciudadano es el vencedor de la guerra del Peloponeso y por esta razón reclama su derecho a una mayor participación en la esfera pública y en el sistema político y encuentra en la educación el medio para lograrlo. De esta forma en la Atenas de Pericles, una ciudad cosmopolita y en crecimiento, el sistema educativo permitía que todo ciudadano libre pueda inscribir a sus hijos y recibir educación, la misma que no era exclusiva de una élite como sí ocurría en otros pueblos griegos, de esta forma, los jóvenes estudiantes podían recibir desde poesía hasta filosofía y participar de los torneos de canto, teatro, danza, gimnasia, asegurando de esta manera que todo hombre libre pueda ejercer sus derechos y deberes ciudadanos (Álvarez, 2016). Por todo ello los ciudadanos de Atenas le daban a la educación una vital importancia y son los sofistas que en ese contexto y momento histórico introducen una forma de educación independiente del Estado, una educación que permitía el desarrollo de las capacidades y habilidades para tener éxito, basada en la relación entre maestro y discípulo y en el uso del libro como método educativo (De Romilly, 1997). Una educación orientada al uso práctico en la vida pública, por consiguiente centrada en lo pragmático, en la mejora de uno mismo y el desarrollo de las capacidades como medio de crecimiento social, siendo de este modo precursores del desarrollo personal. Daban sus enseñanzas ya sea en plazas públicas, en casas particulares o en 
locales que alquilaban para enseñar, se convierten de esta forma en los primeros profesionales de la enseñanza.

\section{¿QUIÉNES FUERON LOS SOFISTAS?}

Los sofistas fueron un grupo de pensadores griegos que aparecen en la segunda mitad del siglo V a.C., no pertenecían a Atenas, eran extranjeros que atraídos por el esplendor cultural y económico de esta ciudad griega, realizan actividades de enseñanza y educación. Los sofistas herederos de la tradición educativa de los poetas, fueron llamados

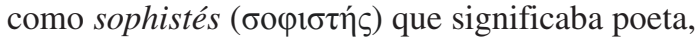
quienes enseñaban, escribían y poseían un conocimiento que impartir ( Guthrie, 2003).

Los sabios sofistas hacen su entrada en la vida social y pública centrados en un conocimiento más pragmático, llevan el conocimiento y la sabiduría a la esfera de la participación pública por medio del arte de la oratoria, y con la fuerza de su discurso son exitosos en el arte de la persuasión y demuestran su éxito en la asamblea y en los debates públicos. Se convierten en los mejores oradores y rápidamente tienen éxito en la asamblea y son reconocidos por su dominio en el arte de convencer. Cualquier ciudadano libre podía gracias a la democracia participar en política y tener éxito, formar parte de las instituciones de gobierno; la habilidad para convencer a un auditorio, a un tribunal o ser elegido públicamente, eran requisitos necesarios para triunfar en la asamblea (Guthrie, 2003).

Con su influencia los sofistas ayudan a modernizar las enseñanzas que debían recibir los jóvenes atenienses permitiéndoles de este modo adaptarse a los cambios sociales y culturales de su época, el movimiento sofista influyó de este modo en la reforma de la antigua educación ateniense centrada en las élites aristocráticas y en el filosofar de problemas abstractos y teóricos, deja atrás su interés por la filosofía centrada en la naturaleza y el cosmos para enfocarse en los temas más pragmáticos ayudando a democratizar la educación y a centrar a la filosofía en lo pragmático y el desarrollo implícito de la cultura del esfuerzo como medio de ascensión social y la mejora de uno mismo, siendo precursores del desarrollo personal ( De Romilly, 1997). El cambio cultural en la sociedad ateniense de la posguerra, permitió el surgimiento de una nueva generación de ciudadanos griegos, más interesada en la participación política y pública, el movimiento sofista facilitó un cambio fundamental en la sociedad ateniense e incluso en el pensamiento socrático (De Romilly,1997). Todo esto llevó a un creciente interés ateniense, sobre todo en sus nuevas generaciones por la participación en la política, los intereses sociales y la vida pública; sin embargo este cambio no estuvo exento de críticas, esta nueva generación también es percibida como inmoral, amante del placer y cobarde, en comparación con la generación anterior vencedora de los persas y ya que a los sabios sofistas se los vincula a esta nueva generación y a todo este movimiento, se los señala como responsables de esta decadencia (Guthrie, 2003). El término sofista ha sufrido durante muchos siglos del estigma social y académico, evolucionando hasta estar asociado al fraude, el engaño, la mentira, teniendo durante todos estos años una connotación negativa, que empezó desde Platón, quien en sus famosos diálogos los acusa de forma despectiva de demagogos, que buscan agradar e influir a otros, que aparentando educar venden su técnica retórica convirtiéndose de esta forma en mercaderes del conocimiento, ávidos de ganar dinero permaneciendo a la caza de jóvenes nobles y adinerados, hijos de las élites, que les pagan por sus enseñanzas en el arte de la retórica, la argumentación y la persuasión mediante la palabra para que puedan tener éxito en convencer y seducir a su auditorio (Álvarez, 2016). De esta forma los sofistas se enfocan en la educación de los hijos de las familias ricas de Atenas, para ello llegaron a alquilar algunos locales en donde brindaban sus enseñanzas y debido a que cobraban por sus lecciones fueron duramente criticados, el cobrar por enseñar escandalizó a ciertos sectores de la sociedad ateniense, el mismo Sócrates los criticó por aceptar dinero al impartir sus conocimientos, consideraba que estarían limitando su libertad, ya que la sabiduría era algo que debería ser compartido liberalmente entre aquellos a quienes se estima (Guthrie, 2003). Los sofistas también eran expertos en geometría, artes y filosofía centrándose en la reflexión del hombre y su participación en la vida pública desde una perspectiva pragmática, convirtiéndose de este modo en desarrolladores de capacidades: toda persona que recibía sus enseñanzas y era educado por ellos podía estar en condiciones de desarrollar sus capacidades en el arte de la retórica, la oratoria y la persuasión mediante la palabra. Fueron muy populares debido a que demostraban sus capacidades y pericia en el arte de la retórica, pudiendo prestar sus servicios a cualquier persona, cualquier ciudadano ateniense, independiente de su condición, que pudiera pagar por sus servicios y que necesitara 
prepararse para ser exitoso en la vida pública y en la política.

\section{EPISTEMOLOGÍA SOFISTA : RELATIVISMO Y ESCEPTICISMO}

Los presocráticos se centraban en el estudio de los fenómenos de la naturaleza y los explicaban a partir de leyes inmutables, que eran válidas para todos los pueblos y culturas, en cambio para los sofistas, quienes eran más cosmopolitas y estaban acostumbrados a viajar de un pueblo a otro enseñando el arte de la retórica, se percatan que los hechos y costumbres pueden cambiar de un pueblo a otro, incluso drásticamente, encontrando muchas diferencias culturales (Guthrie, 2003). Descubren que en el plano terrenal y humano existen diferencias en creencias, percepciones y culturas, esto les sirve para realizar un distingo entre las leyes de la naturaleza y las leyes que se aplican a los hombres, en una concepción escéptica y relativista, que distingue el orden natural y el orden cultural, siendo este último producto más bien del consenso y acuerdo entre los seres humanos (De Romilly, 1997). Para el movimiento sofista, a diferencia de los socráticos, la epistemología y la teoría del conocimiento están influenciados por un escepticismo y relativismo, para ellos es imposible llegar a una verdad absoluta, ya que no es posible tener una certeza absoluta de las cosas, desde la perspectiva sofista más pragmática, todos los puntos de vista de la realidad pueden ser válidos y también inválidos, correctos o incorrectos, no existe una verdad absoluta, ya que esta es influenciada por las circunstancias y condiciones en la que es formulada (Arduini, 2004). Abandonan la idea de una realidad absoluta e inmutable, desde la perspectiva sofista, no existe una realidad absoluta sino relativa, al igual que las leyes humanas son relativas y modificables, el conocimiento es relativo y requiere del sujeto cognoscente (Guthrie,2003). El mismo Protágoras exhibiendo el pragmatismo que caracteriza a los sofistas, menciona que si una persona al probar un pan lo cataloga como desagradable y otra persona al probar el mismo pan lo considera agradable, no habría forma de conocer el verdadero sabor del pan al margen de las opiniones dadas por los protagonistas, por lo que debe buscarse la opción más práctica y útil (Solana, 2000).

Para los sofistas no puede existir conocimiento sin sujeto, como lo menciona Protágoras "El hombre es la medida de todas las cosas, de las que son en cuanto son y de las que no son en cuanto no son"
(Protágoras y Gorgias, 1984). La relación entre el sujeto cognoscente y el objeto conocido pasa por privilegiar el papel del sujeto en la construcción de la realidad (Boodin, 1911). Desde esta perspectiva sofista se privilegia el conocimiento práctico sobre el conocimiento teórico, se introduce el acierto o desacierto, éxito o fracaso en lugar de verdad o error (Guthrie, 2003). Debido a ello su influencia en la terapia breve estratégica es importante, como menciona Protágoras: "Dispone en orden tres cosas capitales: una y primera, que nada existe: segunda, que aunque exista, es incomprensible para el hombre y tercera, que aunque sea comprensible, ciertamente es incomunicable e inexplicable al vecino" (De Romilly, 1997). No se puede conocer la realidad sin actuar sobre ella, solo puedes conocer desde la experiencia a partir de un conocimiento empírico y ese es precisamente el conocimiento de la modificación de las cosas desde la experiencia (Segal, 1994). Todo esto llevó a que los sofistas se centraran más que en estudiar lo abstracto y la filosofía de la naturaleza a una filosofía de la vida cotidiana, el pensamiento filosófico da un giro y pasa de preocuparse y estar centrado en la naturaleza a pensar en la polis, ya no interesa tanto filosofar en torno al cosmos y las estrellas sino centrarse en la vida cotidiana, en los saberes prácticos que sirvan justamente para el día a día, para lo cotidiano. Los sofistas cobran mayor relevancia, porque están relacionados justamente con ese giro y cambio de foco a lo público, siendo más pragmáticos y relacionándose con temas cotidianos como la problemática de la ciudad, su organización, su legislación, la distribución de poder, la participación democrática, entre otros. El escepticismo sofista que los caracterizó, es un rechazo a la postura de los presocráticos sustituyendo a los poetas y rapsodas depositarios del saber, aportando enormemente en el desarrollo de una nueva sociedad ateniense por medio de la enseñanza y su pensamiento filosófico, de esta forma el desarrollo de capacidades y potencialidades de los jóvenes que eran formados por ellos, permitiendo la movilidad y crecimiento social promoviendo la cultura del desempeño y la meritocracia como medio de ascensión social (Delgado, 2014). Aportando de esta manera a consolidar la democracia ateniense, por tanto más allá de los aportes en la retórica, la educación, la gramática, el desarrollo humano, la filosofía, la democracia y el cambio cultural también aportaron en el desarrollo de la moderna psicoterapia y la concepción epistemológica y la praxis clínica. 
TERAPIA BREVE ESTRATÉGICA, PERSUASIÓN Y RETÓRICA SOFISTA

Cuando hablamos de la terapia breve estratégica podemos entender cómo mediante el lenguaje la figura del psicoterapeuta es capaz de de-construir una realidad enferma para construir una nueva realidad más funcional (Ceberio y Watzlawick, 2006). Buscando generar bienestar, desarrollando el potencial y proveyendo salud emocional a otro ser humano, que construyó en el lenguaje una prisión invisible que le generó malestar, dolor y sufrimiento. Nuestro lenguaje a partir de su surgimiento nos ha acompañado como especie moldeando nuestra vida y nuestra ontogenia, los sistemas lingüísticos que utilizamos, así como las construcciones y modelos culturales en los que se desarrollan esos sistemas lingüísticos, nos permiten construir una realidad y actuar en el mundo a base de dicha percepción de la realidad (De la Cruz, 2008).

En la antigua Grecia, era conocida la habilidad de los sofistas de utilizar la palabra por medio de la persuasión y la retórica, esta capacidad no solo se restringía al uso político, por tanto no solo se aplicaba en la oratoria y el debate propio de la plaza pública y el ágora, sino también la usaba con maestría y éxito en la salud, en la curación de las denominadas enfermedades del alma (Laín, 2005). De esta forma los sofistas usaban la palabra como medio de curación de enfermedades, mediante el uso de la persuasión, la retórica y también de la estrategia. Los sofistas no solo dominaban el arte de la persuasión sino que también podían usarla para curar personas, entre los primeros usos de la palabra con fines psicoterapéuticos, se menciona que Antifonte consolaba y aliviaba mediante la palabra a los enfermos que padecían de pesar y aflicción, mencionándose que la palabra como el fármaco no solo domina la voluntad sino que puede modificar

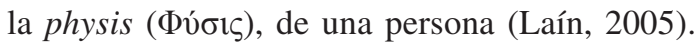
Antifonte fue el precursor de los terapeutas estratégicos, quien obtuvo una gran fama y enorme éxito en la curación de los males físicos por medio de la palabra (Nardone, 2013). El poder de la persuasión puede sanar aún más a un enfermo, la persuasión puede unirse a los poderes del fármaco para curar la

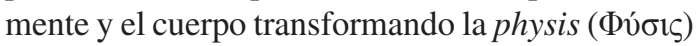
alterada de sus pacientes (Laín, 2005).

Para Gorgias quien al atender a un enfermo de los denominados males del alma, lo ayuda modificando el nomos (vó $\mu$ o $)$ de esta persona en el que se encuentra, un nomos (vó $\mu$ ○) que es entendido como lo legal y convencional para esta persona, su forma de ver e interpretar su realidad, que la lleva a tener una opinión particular, una forma de percibir el mundo, pero que a la vez le genera un estado que le causa aflicción y sufrimiento, un estado del que no puede salir por sus propios medios (Quiñonez, 2017). El sofista Gorgias, mediante la persuasión logra sacar a la persona del nomos que le causa aflicción y sufrimiento para llevarlo a otro nomos (vó

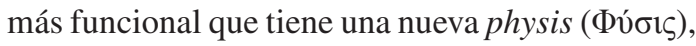
un estado natural y genuino de la vida humana (Quiñonez, 2017). Gorgias, convencía mediante el uso refinado de las palabras, el uso metafórico de las argumentaciones, su habilidad retórica era tal, que se dice que era capaz de responder a cualquier pregunta (Nardone, 2013). En cambio Protágoras se había especializado en la técnica de hacer que sus interlocutores llegaran a contradecirse y esto lo hacía mediante preguntas y comentarios que llevaban a sus interlocutores a una contradicción de sus propios argumentos, logrando que cambien espontáneamente de postura (Nardone, 2013). Ese nomos que usaba Gorgias, es la percepción de nuestra realidad que está pautada por nuestro lenguaje, por las construcciones lingüísticas que utilizamos para describirla y representarla y por la influencia de los patrones culturales y educativos en los que hemos sido criados, nuestra cultura, nuestros valores que tenemos del mundo influyen en nuestra percepción del mundo y nuestra relación con él y determinarán nuestro actuar en él. Los sofistas hacían uso tam-

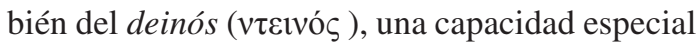
que acompañada de su habilidad persuasiva y de razonamiento, hacía referencia a una habilidad en el uso de estratagemas (Guthrie, 2003). La aplicación por parte de los sofistas de la palabra persuasiva acompañada del deinós ( $v \tau \varepsilon ı v o ́ \varsigma$ ) podía ser usado en la curación exitosa de las enfermedades del alma, acabando con el miedo, produciendo la alegría o intensificando la compasión (Quiñones, 2017). La habilidad de los sofistas de poder modificar el nomos de una persona por medio de la persuasión, acompañándola del deinós ( $v \tau \varepsilon ı v o ́ \varsigma)$, del uso adecuado de estratagemas para poder lograr el bienestar de las personas, está íntimamente relacionado con los terapeutas estratégicos modernos, quienes son hábiles en el uso de la comunicación terapéutica persuasiva acompañada del uso de estratagemas (Nardone, 2013). De este modo, a partir de esta combinación, del nomos y el deinós ( $(v \varepsilon ı$ ós) del terapeuta, quien desde una perspectiva 
distinta y mediante construcciones lingüísticas que enriquecen el modelo del mundo del consultante, permitiendo y facilitando el cambio, son capaces de acompañarlo a experimentar nuevas formas de percibir su realidad.

\section{INFLUENCIA SOFISTA EN LA PRAXIS CLÍNICA DE LA TERAPIA BREVE ESTRATÉGICA DE GIORGIO NARDONE}

En la praxis clínica de la terapia breve estratégica cuando analizamos la relación terapéutica entre el terapeuta y su consultante, lo que el psicoterapeuta en tanto observador y ser humano percibe y construye producto de su realidad cultural, familiar, histórica, educativa, es el resultado de los modelos que utiliza y los mecanismos de percepción que este emplea, de esta forma la teoría que utiliza para aproximarse a la realidad influye en lo observado (Nardone y Balbi, 2018). Los aspectos culturales de dicho terapeuta están presentes tanto en la observación que realiza como en la teoría que utiliza para interpretarla. Desde esta perspectiva el psicoterapeuta en tanto observador no puede captar una realidad objetiva y verdadera, solo puede aproximarse a ella, por esta razón el conocimiento psicoterapéutico que este psicoterapeuta-observador elabore del mundo y de su consultante, no es la representación del mundo real tal y como es, de un mundo objetivo, no es la representación de la verdad absoluta, es solo una aproximación a esta, es la representación de un mundo interpretativo particular, un mundo que existe íntimamente relacionado con el psicoterapeuta en tanto observador que lo observa y de la teoría particular que este psicoterapeuta posee y que le permite entender la realidad de su consultante, es decir, no existe una representación del mundo independiente del observador en tanto persona cognoscente y de sus procesos de construcción de la realidad (De la Cruz, 2013).

Cualquier fenómeno que el psicoterapeuta-observador intente conocer no puede existir independiente de sus propios modelos teóricos, culturales, procesos cognitivos y mecanismos perceptivos (Nardone y Watzlawick, 2018). Desde esta perspectiva de la terapia breve estratégica, siguiendo una tradición sofista y a diferencia de un enfoque tradicional y newtoniano, en donde se concibe que el conocimiento y la interpretación de la realidad es una representación real de un mundo objetivo, de un único universo que existe independiente del observador, lo que llevaría a suponer de que el terapeuta tiene la verdad absoluta, el poder y el conocimiento cabal y preciso de su consultante y por tanto a partir de este conocimiento real y absoluto de su consultante y de su padecimiento, es capaz de intervenir y tratarlo. De esta forma la terapia breve estratégica, bajo una tradición constructivista de influencia sofista y escéptica, considera que no podemos captar el mundo exterior más que solo por medio de nuestros sentidos, de los que tenemos imágenes engañosas (Von Foerster, 1991). Los psicoterapeutas como seres humanos y en tanto observadores y sujetos cognoscentes no pueden describir una realidad independiente de ellos mismos, es decir, solo pueden conocer lo percibido y construido por ellos mismos (Nardone, 2020). Ya que los seres humanos pueden conocer solo lo que ellos mismos han hecho, por esta razón no se puede conocer una realidad independiente del observador (Von Glasersfeld, 2014). Por tanto para la terapia breve estratégica en la relación terapéutica, el conocimiento así construido no representa en ningún caso una realidad objetiva, ni una descripción real del objeto percibido, en este caso del consultante, sino es solo una aproximación, es el producto y reflejo de sus particulares procesos de percepción y de construcción de la realidad del terapeuta. La teoría del observador nos permite concebir el conocimiento como un proceso autorreferencial y autorregulatorio y nos abre el camino para abandonar la concepción de que el conocimiento es una representación objetiva, real y verídica del mundo y de los fenómenos de la naturaleza. La teoría que el terapeuta tiene y con la que describe el mundo, va a influir sobre su actuar y proceder en el espacio terapéutico. La teoría del terapeuta va a dictar de forma determinista cómo debemos de actuar y proceder y qué debe describir respecto del fenómeno observado (Nardone, 2010). El enfoque estratégico de esta forma representa el paso de un tipo de conocimiento positivista y determinista que pretende describir con certeza la realidad de las cosas, que pretende conocer y describir en forma absoluta al consultante y su padecimiento, para pasar a un conocimiento operativo, que busca y nos permite manejar la realidad funcionalmente (Nardone, 2000). A causa de que son las teorías las que determinan lo que debemos observar, la terapia breve estratégica rechaza cualquier teoría que establezca a priori estrategias o intervenciones (Nardone y Portelli, 2017). Se evita definir la naturaleza de las 
cosas, intentamos evitar un modo de intervención universal, es siempre la solución la que se adapta al problema y no al revés, la lógica estratégica se adapta a su objeto de estudio (Nardone y Portelli, 2017). De esta forma al igual que los sofistas que en su momento pasan de centrarse en la filosofía de las leyes inmutables deterministas para hacer filosofía de lo pragmático "el saber cómo". El moderno terapeuta estratégico busca un conocimiento más operativo, pragmático y funcional, que se enfoca en el proceso de cambio y en la observación de la persistencia del problema y no en sus causas, se enfoca más en cómo funciona y en cómo podemos hacer para solucionarlo (Nardone, 2010). Desde lo constructivista, existen múltiples realidades subjetivas como observadores existen, no existe un solo universo sino un multiverso, no existe ontológicamente una verdadera realidad, la realidad es el producto de la perspectiva, los instrumentos del conocimiento y el lenguaje por el que percibimos y comunicamos (Nardone y Portelli, 2017). Se abandona la tesis de que existe un conocimiento científicamente verdadero, se buscan los caminos del conocimiento y actuación más funcionales denominado por Von Glasersfeld como "conciencia operativa" (Von Glasersfeld, 2014). Al igual que los antiguos sofistas, un terapeuta estratégico no busca el porqué de las cosas, ni busca descubrir las leyes que gobiernan la naturaleza, tampoco se interesa en encontrar meticulosamente en el pasado del consultante las causas del problema o del padecimiento, se centra más bien en los aspectos pragmáticos, en cómo funcionan las cosas y en cómo hacerlas funcionar mejor para brindar bienestar al consultante de manera más eficiente y eficaz; estos modernos sofistas se basan en una lógica estratégica centrada en objetivos y adaptan su intervención a las características específicas de cada problema. De este modo la psicoterapia breve estratégica busca incrementar la conciencia operativa, eso involucra la transición de un conocimiento como representación de la realidad objetiva a un conocimiento como representación de la realidad más adaptada (Von Glasersfeld, 2014) y eso involucra dejar de centrarse en la búsqueda de las causas que generaron el trastorno y concentrarse en el manejo estratégico de la realidad que nos rodea con el fin de alcanzar los objetivos, el conocimiento operativo evita adoptar posiciones deterministas. Es un conocimiento más adaptativo que permite orientar nuestro método en el cambio para que se adapte a nuestros objetivos en armonía con nuestro mundo perceptivo.

\section{EL SISTEMA TERAPÉUTICO}

El psicoterapeuta no se encuentra aislado de su consultante, no es un observador externo, ni el consultante un ente pasivo que recepciona pasivamente las intervenciones que el terapeuta tenga a bien realizar, por el contrario, ambos construyen activamente el proceso terapéutico y son responsables de sus resultados, ya que desde el punto de vista cibernético y constructivista el consultante hace terapia al terapeuta tanto como el terapeuta la realiza con el consultante, "la roca esculpe al escultor tanto como este a la roca" (Keeney, 1994). Todo lo que diga el terapeuta en tanto observador respecto de su consultante, dice más del terapeuta y de su teoría, cultura o escuela que del consultante, el terapeuta nunca podrá tener una descripción real y completa del consultante, tan solo una aproximación a esa realidad terapéutica (Nardone, 2020). Cuando el psicoterapeuta destaca y hace distinciones, en tanto observador que es, se convierte en responsable de sus propias construcciones, por tanto pasa de llevar el control, de intervenir desde el exterior a reflexionar y a co-construir la realidad terapéutica, de ahí que la realidad del terapeuta es una construcción del observador que él construye en función al modelo que pregona y que practica; eso nos lleva a entender que la relación terapéutica es una relación de aprendizaje, de cambio, de reflexión y evolución conjunta entre consultante y psicoterapeuta (De la Cruz, 2003). ambos son co-responsables del éxito o fracaso del proceso terapéutico, ambos generan y construyen en la relación a lo largo de cada encuentro, algo superior a ellos, un todo sinérgico llamado sistema terapéutico, un encuentro humano entre seres humanos, de respeto, de legitimación y de amor.

\section{CONCLUSIONES}

En el pensamiento sofista al igual que en la terapia breve estratégica se plantea que no podemos describir una realidad independiente del observador, quien solo puede conocer lo percibido y construido por él mismo. Ambos enfoques conciben que el conocimiento no es en ningún caso una representación real, objetiva y verídica de la realidad.

La praxis clínica de la terapia breve estratégica incorpora todo lo anterior a la relación terapéutica, el terapeuta por tanto no puede en ningún caso tener 
una descripción real, completa y objetiva de su consultante, tan solo una aproximación, mediada por su particular proceso de percepción y construcción de la realidad . Siendo este conocimiento un proceso autorreferencial y autorregulatorio, la descripción de la realidad por parte del terapeuta depende en gran medida de la teoría e influye en lo que el terapeuta observa. En la terapia breve estratégica se deja la certeza y la objetividad para pasar a un conocimiento operativo que busca un manejo funcional de la realidad. Reconocen al igual que los sofistas que existen múltiples realidades subjetivas como observadores existen. De esta manera la realidad solo se puede conocer desde la experiencia, solo desde un conocimiento empírico, por tanto, solo se puede conocer al consultante en tanto empezamos a interactuar con él, un conocimiento desde la experiencia, conociendo la realidad y actuando con ella, con un claro enfoque pragmático, operativo $\mathrm{y}$ funcional. 


\section{REFERENCIAS}

Arduini, S. (2004). Gorgias o de la ideología. Escritura y Pensamiento, 7(14), 9-25.

Álvarez, L. (2016). La función propedéutica del sofista y la emergencia del filósofo. División, dialéctica y paradigmas en el diálogo Sofista. Arete. Revista de Filosofía, 25(2), 337-366.

Boodin, J. (1911). From Protágoras to William James. The Monist, 21(1), 73-91. http://www.jstor.com/stable/27900296.

Ceberio, M. y Watzlawick, P. (2006). La construcción del universo. Herder.

De la Cruz, R. (2003). Drogodependencias. Familia, terapia y epistemología. Devida.

De la Cruz, R. (2008). Violencia intrafamiliar. Enfoque sistémico. Trillas.

De la Cruz, R. (2013). El docente como orientador. Estrategias para el manejo de problemas familiares y sociales. Trillas.

Delgado, D. (2014). Los sofistas al pancracio: Lenguaje y agonismo para la transformación de los valores ciudadanos. Valenciana , 7(13), 59-81.

De Romilly, J. (1997). Los grandes sofistas en la Atenas de Pericles. Seix Barral.

Guthrie, W. (2003). Historia de la filosofía griega. Gredos.

Keeney, B. (1994). Estética del Cambio. Paidós.

Laín, P. (2005). La curación por la palabra en la antigüedad clásica. Anthropos.

Nardone, G. (2000). Terapia breve estratégica. Pasos hacia un cambio de percepción de la realidad. Paidós.
Nardone, G. (2010). Problem solving estratégico: El arte de encontrar soluciones a problemas irresolubles. Herder.

Nardone, G. (2013). El arte de la estratagema. Cómo resolver problemas difíciles mediante soluciones simples. Herder.

Nardone, G. y Balbi, E. (2018). Surcar el mar a espaldas del cielo: lecciones sobre el cambio terapéutico y las lógicas no ordinarias. Herder.

Nardone, G. y Portelli. C. (2017). Conocer a través del cambio: La evolución de la terapia breve estratégica. Herder.

Nardone, G. y Watzlawick, P. (2018). Terapia breve: filosofía $y$ arte. Herder.

Nardone, G. (2020). Miedo, pánico, fobias: La terapia breve. Herder.

Protágoras y Gorgias (1980). Fragmentos y testimonios. Hyspamérica.

Quiñonez, B. (2017). La retórica de Gorgias y el placer de la palabra. Limes, 27, 77-90.

Segal, L. (1994). Soñar la realidad. El Constructivismo de Heinz Von Foerster. Paidós.

Solana, J. (2000). El camino del ágora, filosofía política de Protágoras de Abdera. Prensas Universitarias de Zaragoza.

Von Foerster, H. (1991). Las semillas de la cibernética. Gedisa.

Von Glasersfeld, E. (2014). El constructivismo radical o la construcción del conocimiento. En P. Watzlawick y G. Nardone. (Eds.). Terapia breve estratégica. Pasos hacia un cambio en la percepción de la realidad (pp. 39-49). Paidós. 